\title{
Upper Limit of Normal for Serum Alanine Transaminase in Healthy Population
}

\author{
Beuy Joob $^{1} \cdot$ Viroj Wiwanitkit ${ }^{2,3}$
}

Received: 25 September 2017/ Accepted: 16 October 2017/Published online: 24 October 2017

(C) Association of Clinical Biochemists of India 2017

Dear Editor, we read the recent publication on "Upper Limit of Normal for Serum Alanine Transaminase (ALT)" with a great interest. Mohen et al. [1] reported that "The mean ALT in men and women were $21.87 \pm 2.9$ (97.5th percentile $28 \mathrm{U} / \mathrm{L}$ ) and $19.35 \pm 3.3$ (97.5th percentile 24 U/L) respectively." and concluded that "There is a need to re-consider ALT levels in our population for better detection of individuals at risk for liver disease [1]." We would like to share ideas and experience on this topic. First, the healthy population in this study might not a disease free population. The statistical confirmation for the normal distribution of the ALT results is required for setting any normal reference value. In our experience from Thailand, where there is a high rate of silent hepatitis virus infection, the reference value derived from the study of healthy people, without screening for hepatitis B carrier, is high
[2]. If the reference value is intended for using for detection of individual risk for liver disease, exclusion of the case with underlying silent hepatitis carrier status is needed.

\section{Compliance with Ethical Standards}

Conflict of interest None.

\section{References}

1. Mohan P, Sundar V, Bhaskar E, Anthony S. Estimation of upper limit of normal for serum alanine transaminase in healthy South Indian population. Indian J Clin Biochem. 2017;32:337-42.

2. Wiwanitkit V, Tangdhanakanond K. Clinical chemistry laboratory references parameters. Chula Med J. 2003;47:97-100.
Beuy Joob

beuyjoob@hotmail.com

Sanitation 1 Medical Academic Center, Bangkok, Thailand

2 Hainan Medical University, Haikou, China

3 Dr. DY Patil University, Pune, India 